García, V M., Meza, L. C., y Pedraza, F. (2018). Desarrollo de la capacidad dinámica de absorción de conocimiento entre empresas familiares y no familiares de Bucaramanga, Colombia. Revista Lebret, 10, 89-109 - ISSN Versión impresa: 2145-5996 - ISSN Versión Web on line: 2357-5468 • https://doi.org/10.15332/rl.v0i10.2199

\title{
Desarrollo de la capacidad dinámica de absorción de conocimiento entre empresas familiares y no familiares de Bucaramanga, Colombia ${ }^{1}$
}

\author{
Development of the dynamic capacity to absorb \\ knowledge between family and non-family businesses \\ in Bucaramanga, Colombia
}

Victor Manuel Garcia Valenzuela Lucelly Carolina Meza Ariza Fabiola Pedraza Bautista

\section{Resumen}

La capacidad dinámica de absorción del conocimiento (CDAC) es definida por Cohen Levinthal (1990) como la habilidad de las empresas para reconocer nueva información y lograr asimilarla con el conocimiento ya existente, para utilizarla con fines comerciales mediante la generación de ventajas competitivas y así generar valor al cliente. En una comparación entre empresas familiares vs no familiares se determina cuál de ellas genera más CDAC y a su vez se explica la relación entre sus dimensiones mediante el método de $\mathrm{MCO}$ aplicando 3 modelos de regresión lineal para cada categoría de empresas. Los resultados indicaron que con un $95 \%$ de confianza las empresas familiares tienen mayor capacidad de explicación y una mayor suma en los cálculos de los coeficientes de las variables introducidas en los modelos, por tanto reflejan más capacidad de absorción.

\section{Palabras clave}

Capacidad de absorción, ventajas competitivas, capacidades dinámicas.

\section{Códigos de clasificación JEL: D21, D83, M21}

\begin{abstract}
The dynamic capacity of absorption of knowledge (CDAC) is the definition of Cohen and Levinthal (1990) as the ability of companies to recognize new information and assimilate knowledge and use to use it for commercial purposes by generating competitive advantages to generate value to the client. In a comparison between family and non-family businesses, it is determined how they are explained more frequently. It is a relation between its dimensions using the OLS method, applying 1 linear regression models for each type of company and each dimension. The results indicate a $95 \%$ confidence in family businesses that have a greater capacity for explanation and a greater sum in the calculations of the coefficients of the variables introduced in the models.
\end{abstract}

\section{Keywords}

Absorption capacity, competitive advantages, dynamic capacities.

\section{Artículo de investigación.}

2 Instituto Tecnológico de Sonora México. Correo electrónico: victorgarciavzl@gmail.com. Código ORCID: 0000-0002-6109-2135

3 Universidad Santo Tomás Seccional Bucaramanga. Correo electrónico: lucelly.meza@ustabuca.edu.co. Código ORCID: 0000-0002-9373-0306

$4 \quad$ Universidad Santo Tomás Seccional Bucaramanga. Correo electrónico: fabiola.pedraza@ustabuca.edu.co.Código ORCID: 0000-0002-5983-1904 


\section{Introducción}

Las empresas familiares logran un papel importante en la economía de un país, al evaluar su participación en el tejido empresarial es relevante su distribución en algunos países; en Estados Unidos corresponden al 96\%, en Italia al 99\%, en España al $71 \%$ y en Colombia oscila entre el 70 y 75\% según Gaitán \& Castro (2005). El concepto de empresa familiar no presenta una definición generalizada, hay quienes consideran que son aquellas organizaciones donde quien ejerce control y realiza el proceso de toma de decisiones es el mismo dueño, otros opinan que es cuestión de distribución de la propiedad o la forma de gobierno que tiene la unidad de negocio (Serna, 2006).

Ronquillo (2006) destaca el conocimiento profundo del negocio de familia, la flexibilidad en la forma de trabajar y administrar el tiempo y el sentimiento de pertenencia y compromiso como ventajas comparativas frente a otro tipo de organizaciones. Sin embargo, otros autores identifican el alto grado de rigidez de los métodos empresariales, la alta centralización de la toma de decisiones (Poza, 2004), la sucesión en el mando y la convivencia en un mismo tiempo y espacio de los ámbitos familiar y empresarial (Gallo, 2004) como grandes desventajas del modelo de negocio.

En el mismo caso de las economías, las empresas también entran dentro de una nueva era de tecnología, información y conocimiento, por lo tanto, en lo que se basa el nuevo desarrollo empresarial está en el aumento y progreso del conocimiento productivo. Todos aquellos conocimientos productivos pueden ser asociados a las capacidades dinámicas que desarrollan las empresas y se ven reflejados en el nivel de crecimiento y riqueza de estas.

Actualmente, las empresas enfrentan retos de supervivencia cada vez más complicados en el mercado o sector donde se empeñan, según Confecámaras (2016) en Colombia la tasa de mortalidad empresarial de los nuevos emprendimiento es de $70 \%$, es decir, que de cada 10 empresas que son constituidas en Colombia, solo tres de ellas logran sobrevivir en el mercado. En Colombia la mayoría de las empresas están clasificadas como de tamaño micro y pequeñas empresas, que con gran dificultad logran sobrepasar los primeros 3 años de vida en el mercado, generalmente este tipo de empresas carece de una habilidad de reaccionar frente a los cambios que puedan presentarse en el entorno que se encuentra y que puedan afectar el negocio según Celeita (2016).

Esto puede determinarse por la falta de competitividad en las empresas, no arriesgarse ni involucrarse con un gran impacto en el sector y por ende desaparecer del mercado. La innovación es una de las herramientas empresariales para combatir la mortalidad empresarial y sobrevivir a la brecha de mortandad corporativa y es la misma innovación producto de la CDAC. 
La capacidad de absorción es definida por Cohen \& Levinthal (1990) como una habilidad de las organizaciones para reconocer nueva información, de tal manera que logren asimilarla con el conocimiento ya existente, lo cual le permite aplicarla con fines comerciales. Máynez, Cavazos y Nuño (2012) argumentan que la capacidad de absorción les brinda a las empresas, a través de la transferencia del conocimiento, las capacidades para desarrollar ventajas competitivas en el mercado.

Según Díaz, Aguiar y De Saá (2006) la economía se caracteriza por una fuerte competencia, considerando el conocimiento como un recurso valioso para las empresas, debido a que la creación y sostenibilidad de las ventajas competitivas de las organizaciones no se explica por la posición en el mercado por sí sola, sino por la dificultad de replicar los activos intangibles y la forma en la cual estos se desarrollan dentro de la empresa. En los últimos años se ha visto incrementada la percepción de los empresarios de la importancia por medir los resultados obtenidos con base en las inversiones realizadas en activos de conocimiento intangibles.

Flor, Oltra, \& García (2011) denominan la capacidad de absorción del conocimiento como "El concepto de capacidad de absorción que recoge la habilidad de una empresa para reconocer el valor de información nueva, externa a la empresa, para asimilarla y aplicarla con fines comerciales" (p. 69).

Al ser tan importante la CDAC para las empresas, aquí radica la relevancia del impacto de la investigación al estudiar cómo las dimensiones de la capacidad de absorción se relacionan entre sí, y a su vez permite dar respuesta al interrogante ¿qué tipo de organización tiene mayor desarrollo de la capacidad de absorción, las empresas familiares o las no familiares?, para el estudio se tomó en cuenta los dos sectores más participativos en el PIB de Bucaramanga, Santander, Colombia.

\section{Antecedentes}

A continuación se muestran diferentes antecedentes acerca de la conceptualización de la CDAC así como de sus dimensiones con la finalidad de profundizar la comprensión e indagar más en el tema. La revisión de literatura permite apreciar la variedad de artículos de revisión bibliográfica del concepto de CDAC, además la mayoría de los autores asocian la CDAC como insumo necesario para las empresas que desean desarrollar la capacidad para innovar, pese a lo anterior, se identifican pocos estudios empíricos sobre la CDAC y en especial en Colombia. La capacidad dinámica de absorción del conocimiento juega un papel determinante, ya que es mediante ella que se logra la incorporacion de nuevos conocimientos externos que permiten mejorar los bienes y servicios, ya sea en cuanto a calidad, textura, periodo de vida, utilidad, entre otras. Esta capacidad dinámica, por parte de los nuevos empresarios, podría fomentar el crecimiento económico, además el desarrollo de esta capacidad implica identificar, asimilar y explotar nuevos métodos productivos, administrativos 
y financieros, causando un pleno crecimiento laboral internamente en la empresa como económico (Lane, Koka y Pathak, 2006).

Díaz, Aguiar y De Saá (2006) argumentan que la CDAC tiende a incrementar la innovación en las empresas, explicando así las diferencias entre los resultados de empresas del mismo sector, los autores proponen que las diferencias en los resultados provienen de la inversión realizada en activos tangibles. Asimismo, Franco y Urbano (2010) en su investigación identificaron y analizaron las causas que determinan el éxito empresarial en las pymes de Colombia, haciendo referencia a los factores internos y externos del entorno empresarial, basándose de igual manera en primera instancia en la Teoría de Recursos y Capacidades. El estudio cualitativo revela los factores causantes del éxito empresarial, los cuales son conocimiento y experiencia del empresario.

La óptima gerencia en las empresas permite que las compañías sean más competitivas al crear e incentivar las redes de conocimiento dentro de la organización, aprovechando de forma maximizada sus capacidades y recursos tangibles e intangibles. Para los autores anteriores la diferencia entre los recursos intangibles que conforman las empresas permite por sí misma generar ventajas competitivas, en contraposición se encuentra el planteamiento de Flor, Oltra, \& García (2011), quienes demuestran que la capacidad dinámica de absorción del conocimiento variará según las estrategias empresariales adoptadas por los empresarios, además determinan que dicha capacidad es desarrollada a través de la habilidad de adquirir-asimilar-transformar-explotar el conocimiento generado, en esta misma corriente se encuentran los autores Máynez, Cavazos y Nuño (2012), en su investigación analizan la influencia de la capacidad de absorción y la cultura organizacional como determinantes para que la transferencia de conocimiento se logre de manera exitosa y eficiente, las investigaciones de los autores anteriores demuestran que las organización para generar ventajas competitivas deben ir más allá de la inversión en capital intangible, siendo relevante la generación de estrategias que permitan la construcción de conocimiento organizacional.

En función a los referentes teóricos anteriormente exhibidos se puede concluir que la CDAC es la capacidad de las organizaciones de identificar nuevo conocimiento en el entorno, comprenderlo de forma que pueda transformar el conocimiento explícito a tácito para desarrollar y apropiar nuevas prácticas empresariales y, finalmente, aplicarlas generando mayor valor en los bienes y servicios comercializados, construyendo así ventajas competitivas sostenidas.

\section{Relevancia de la capacidad dinámica de absorción}

Al desarrollar nuevas redes de conocimiento e innovación se logra crear nuevas ventajas competitivas en las empresas en contraste con la competencia, las cuales pueden ser aprovechadas mediante estrategias empresariales. Esto permite a las em- 
presas desarrollar nuevas propuestas de valor en sus productos, ya que logran obtener mayor demanda potencial; al ser altamente demandados se facilita la comercialización de dichos productos y servicios, por lo tanto la factibilidad de producir bienes y servicios innovadores con calidad se vuelve viable para este tipo de empresas y favorece su permanencia en el mercado (Herrera, Parra, Livscovsky, Ramos y Gallardo, 2017).

Según Global Entrepreneurship Monitor (GEM) en el 2005 la tasa de mortalidad de las empresas en Colombia es una de las más altas en todo Latinoamérica, la cual asciende al casi $11 \%$, este indicador interpreta la necesidad de reformar los modelos de desarrollo empresarial actuales para lograr una mejora en el nivel de sostenibilidad de los empresarios en el mercado (Peña, Bravo, Álvarez, Pineda, 2011).

En concordancia con la teoría, aquellas empresas que obtengan altos niveles de la capacidad dinámica de absorción del conocimiento aplican estrategias que permiten aprovechar la información externa y generar nuevas prácticas que agregan valor a sus bienes y servicios, por lo tanto, la generación de nuevo conocimiento en los procesos productivos permite a las empresas crear ventajas competitivas a través de la diferenciación; la innovación en los productos logra posicionar a las empresas en un entorno más competitivo. Asimismo, una empresa al convertirse altamente competitiva en el mercado y al encontrarse en constante desarrollo de mejora en sus procesos productivos, incentiva el desempeño empresarial de la misma por lo cual le permite permanecer en el mercado de forma exitosa.

\section{Constructo de la capacidad de absorción}

\section{Capacidad dinámica de absorción del conocimiento (CDAC)}

La única forma de crear ventajas competitivas sostenidas es a través del recurso más importante de la empresa: Talento humano (Nanoka y Takeuchi, 1995). Mediante la transferencia de conocimiento productivo táctico surge la creación y acumulación de conocimientos, lo cual permite a la empresa identificar sus recursos internos, que le permiten tener diferentes capacidades empresariales, que están encaminadas a buscar mejores beneficios para la empresa mediante la creación de estrategias basadas en ventajas competitivas, con el objetivo de buscar la diversificación de sus procesos productivos y la implementación de procesos complejos, es decir, surge la innovación en las empresas en la búsqueda de la mejora continua en los procesos productivos, financieros o administrativos (García, Ruiz López y Plascencia, 2018).

La transferencia de conocimiento es una de las etapas de la capacidad dinámica de absorción de conocimiento, la cual es una de las capacidades más importantes del talento humano dentro de una organización (Filgueiras, Castro y Rafull, 2013). La capacidad de absorción es definida por Cohen \& Levinthal (1990) como una habilidad de las organizaciones para reconocer nueva información, de tal manera que 
logren asimilarla con el conocimiento ya existente, lo cual le permite aplicarla con fines comerciales. Máynez, Cavazos y Nuño (2012) argumentan que la capacidad de absorción les brinda a las empresas a través de la transferencia del conocimiento las capacidades para desarrollar ventajas competitivas en el mercado. Gonzáles y Hurtado (2014) mencionan que la capacidad de absorción es una capacidad dinámica que ayuda a desarrollar y explotar el conocimiento externo. La capacidad dinámica de absorción de conocimiento es descrita en la tabla 1 .

Tabla 1. Descripción de las dimensiones de la capacidad de absorción

\begin{tabular}{ll}
\hline \multicolumn{1}{c}{ Dimensión } & \multicolumn{1}{c}{ Descripción } \\
\hline Adquisición & $\begin{array}{l}\text { Se refiere a los esfuerzos realizados en la identificación y adquisición del nuevo } \\
\text { conocimiento. }\end{array}$ \\
Asimilación & $\begin{array}{l}\text { Se refiere a los esfuerzos realizados por analizar, procesar y comprender la nueva } \\
\text { información identificada. }\end{array}$ \\
Transformación & $\begin{array}{l}\text { Se refiere a los esfuerzos realizados por implementar el nuevo conocimiento } \\
\text { y unirlo con el existente en búsqueda de mejorar y desarrollar las rutinas de la } \\
\text { empresa. }\end{array}$ \\
Explotación & $\begin{array}{l}\text { Se refiere a los esfuerzos realizados por perfeccionar las antiguas rutinas y generar } \\
\text { nuevas competencias. }\end{array}$ \\
\hline
\end{tabular}

Fuente. Con base en Flor, Oltra y García (2011).

Estas fases dentro de la capacidad dinámica de absorción logran estimar el comportamiento del proceso de absorción de conocimiento en una empresa. Las cuatro fases se complementan unas a otras, todas son importantes para que la absorción de conocimiento se logre, sin embargo, es en la última (explotación) donde ocurre la innovación en las organizaciones para crear ventajas competitivas sostenidas, las cuales le permitan a la empresa perdurar en el mercado al generar más valor al cliente a través de sus productos o servicios.

\section{Ventajas competitivas}

Las ventajas competitivas son un vehículo para el desarrollo y crecimiento de las empresas en busca del éxito empresarial. Las ventajas competitivas según Porter (1991) citado por Pavez (2000) son aquellas que provienen del valor que generan las empresas para los clientes, justificando así que se pague un excedente en el precio a cambio de beneficios particulares que ofrece el bien o servicio adquirido. Las ventajas competitivas se ven reflejadas en el valor agregado en un bien o servicio, el valor agregado se refleja en los clientes de las organizaciones y los clientes en los beneficios financieros generados por la empresa. Las ventajas competitivas brindan a las empresas la capacidad de adaptarse a las oportunidades y a los distintos cambios que pueden presentarse en el mercado.

Fong (2011) determina que cuando la rentabilidad de una empresa está por encima de la media del sector al que pertenezca, esta organización posee una ventaja 
competitiva, sin embargo, para garantizar su sostenibilidad su diferenciación debe ser única e inimitable. Un principio clave para la existencia de las ventajas competitivas es que las empresas del sector tengan recursos y capacidades diferentes, de esta forma puede diferenciarse el valor agregado generado por las mismas y a su vez obtener beneficios financieros mientras alcanzan el éxito empresarial según Suárez, Ibarra (2002).

La relación de las ventajas competitivas y la CDAC radica en la última dimensión, la capacidad de Explotación, que es donde el conocimiento es implementado para la creación de nuevos productos o servicios, surgen mejoras continuas dentro de la empresa en procesos productivos, administrativos o financieros, la empresa genera valor agregado a sus consumidores, es decir, surge la innovación en las organizaciones en busca de crecimiento y desarrollo empresarial.

\section{Metodología}

\section{Tipo de investigación}

El presente trabajo de investigación tiene un alcance correlacional con un diseño no experimental a razón de que ninguna de las variables analizadas introducidas en el modelo fue manipulada. La información es una base de datos recopilada en un solo momento o tiempo único denominado corte transversal. En relación con los objetivos y el tipo de información se considera un análisis de tipo cuantitativo.

\section{Muestra}

Se tomó una muestra no probabilística y a conveniencia de 68 empresas ubicadas en la ciudad de Bucaramanga que se distribuyen en dos categorías: 34 empresas familiares y 34 empresas no familiares, se tomó la misma cantidad de empresas con el fin de analizar las dimensiones de la capacidad de absorción en cada categoría y realizar una comparación de los resultados.

Las empresas participantes en el estudio corresponden al sector industrial en calzado, confección, panificadoras, muebles, transformación de alimentos; y en el sector servicios en inmobiliario, hotelero, asegurador, financiero y salud.

\section{Materiales}

Se aplicó un instrumento (encuesta estructurada) para recolectar la información de las empresas, la aplicación se realizó a los dueños o cargos administrativos relacionados con talento humano. El instrumento se sometió a una prueba piloto con la participación de 10 empresas para comprobar su calidad y validez. Posteriormente, se procedió a reformar algunas preguntas debido a su lenguaje técnico. En el do- 
cumento se especificó los procedimientos para su utilización y el compromiso de confidencialidad de la información.

La encuesta se conforma de 16 ítems, de los cuales 13 son afirmaciones correspondientes a las dimensiones de la CDAC y 3 son preguntas generales para establecer una caracterización de las empresas que participaron del estudio. Las afirmaciones se analizaron con base en la escala Likert, la cual es una escala psicométrica utilizada para la comprensión de las opiniones del encuestado, se aplicó una escala de 5 niveles, donde 1 es absolutamente en desacuerdo y 5 es absolutamente de acuerdo. En la tabla 2 se muestra la conformación de las afirmaciones de la encuesta en función de las dimensiones de la CDAC.

Tabla 2. Conformación del instrumento

\begin{tabular}{|c|c|c|}
\hline Afirmación & Variable & Autor \\
\hline $\begin{array}{l}\text { La empresa busca constantemente información } \\
\text { relacionada con su industria o negocio. }\end{array}$ & \multirow[t]{4}{*}{ Capacidad de adquisición } & \multirow[t]{4}{*}{$\begin{array}{l}\text { Tzokas, Kim, Akbar; } \\
\text { Al-Dajaniet al., } 2015\end{array}$} \\
\hline $\begin{array}{l}\text { La mayoría de los empleados de esta } \\
\text { organización son profesionales con estudios de } \\
\text { posgrado. }\end{array}$ & & \\
\hline $\begin{array}{l}\text { La Dirección motiva a los empleados para buscar } \\
\text { fuentes de información del sector. }\end{array}$ & & \\
\hline $\begin{array}{l}\text { La organización brinda capacitaciones } \\
\text { constantes a sus empleados. }\end{array}$ & & \\
\hline
\end{tabular}

En esta empresa las ideas y conceptos se

Capacidad de Asimilación Zahra y George, 2002 comunican entre departamentos.

La Dirección fomenta el apoyo entre

departamentos para resolver problemas.

Los jefes incentivan a su equipo de trabajo a compartir sus experiencias y prácticas usadas para resolver inconvenientes presentados en las actividades desarrolladas.

Los empleados vinculan acertadamente el conocimiento existente con nuevas ideas.

Los empleados de esta empresa son capaces de aplicar los nuevos conocimientos en su puesto de trabajo.

Esta empresa incentiva a sus trabajadores a mejorar los procesos existentes.

La Dirección apoya el desarrollo de muestrarios Capacidad de explotación Z Zahra y George, 2002
y prototipos.

Esta empresa tiene la capacidad de adoptar continuamente nuevas tecnologías.

La empresa promueve la investigación y el desarrollo.

Fuente. Elaboración propia. 


\section{Método estadístico}

Se implementaron tres modelos de regresión lineal para cada tipo de empresas (familiares y no familiares) entre cada una de las dimensiones de la CDAC, para conocer la relación entre las mismas, el nivel de dependencia y afirmar la relevancia o existencia consecuente entre las combinaciones. En la figura 1 se muestra la operacionalización de las variables y combinaciones de parejas de los modelos de regresión.

\section{Operacionalización de las variables}

\begin{tabular}{|c|c|c|}
\hline Variable independiente & & Variable dependiente \\
\hline Dimensión: & & Dimensión: \\
\hline Adquisición & vs & Asimilación \\
\hline Asimilación & vs & Transformación \\
\hline Transformación & vs & Explotación \\
\hline
\end{tabular}

Fuente. Elaboración propia.

Se opta por realizar un modelo de regresión lineal simple y no multivariado al ser las dimensiones de la CDAC de carácter cíclicas, por ende, la ocurrencia de la dimensión 4 dependerá de la ocurrencia de la dimensión 3 y la ocurrencia de la dimensión 3 de la ocurrencia de la dimensión 2 y la ocurrencia de la dimensión 2 de la ocurrencia de la dimensión 1, según Cohen y Levinthal (1990). A su vez, se determina la relación entre la secuencia de las dimensiones y si existe la presencia de las 4 en las empresas analizadas. Mediante la metodología de mínimos cuadrados ordinarios (MCO) se minimizó la distancia entre las desviaciones cuadradas de los valores observados respecto a su media. Se eligió el MCO al ser de las metodologías más utilizadas para los modelos de regresión por sus propiedades estadísticas de alto nivel de eficacia en este tipo de investigaciones y estudios.

\section{Resultados}

Consecuente a la tabulación de los datos recolectados mediante el instrumento implementado a empresas familiares y no familiares, se presenta la caracterización de las empresas que participaron en la investigación.

De acuerdo con la distribución de las empresas en los subsectores de estudio se obtienen los resultados relacionados en las figuras 1 y 2 . 
Figura 1. Subsectores empresas no familiares

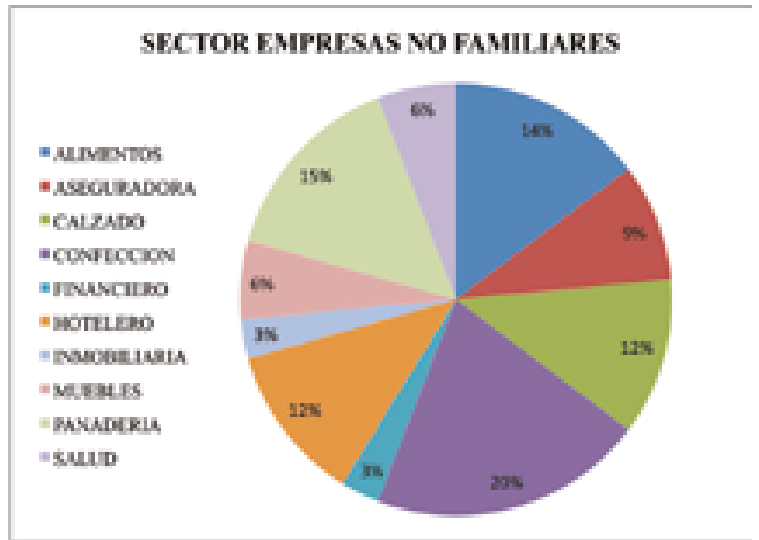

Fuente. Elaboración propia.

Figura 2. Subsectores empresas familiares

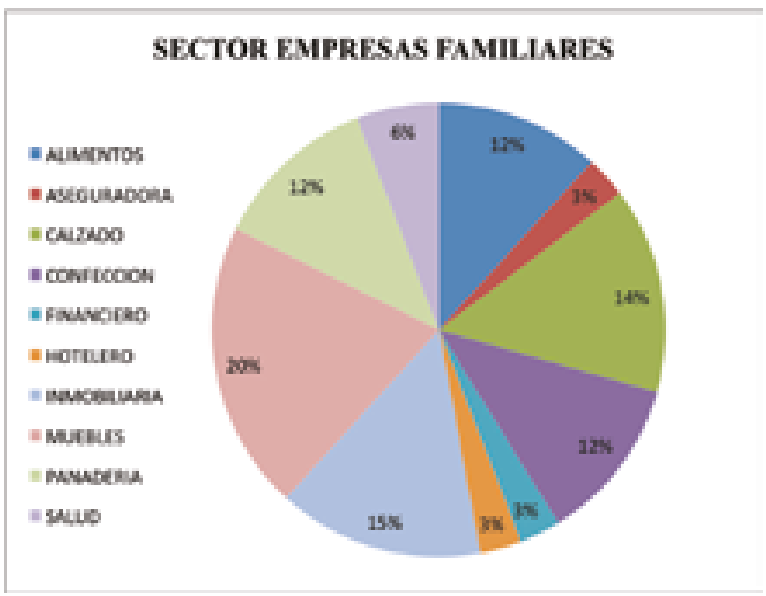

Fuente. Elaboración propia.

Las empresas no familiares que más participaron en la investigación se ubican en los subsectores: confección, panificador, alimentos, hotelero y calzado, representando el $73 \%$ de la muestra, mientras que las empresas familiares destacan los subsectores: muebles, inmobiliarios, calzado, alimentos, confecciones y panificador representando el $85 \%$ de las empresas encuestadas.

Por otro lado, la trayectoria de las empresas encuestadas refleja mayor permanencia en el mercado de las empresas no familiares, teniendo en cuenta que el $38 \%$ de las empresas que participan en la investigación tienen más de 20 años en el mercado 
frente al $21 \%$ de las empresas con la misma trayectoria que conforman la muestra de las empresas familiares, tal como se puede observar en las figuras 3 y 4 .

Figura 3. Trayectoria empresas no familiares

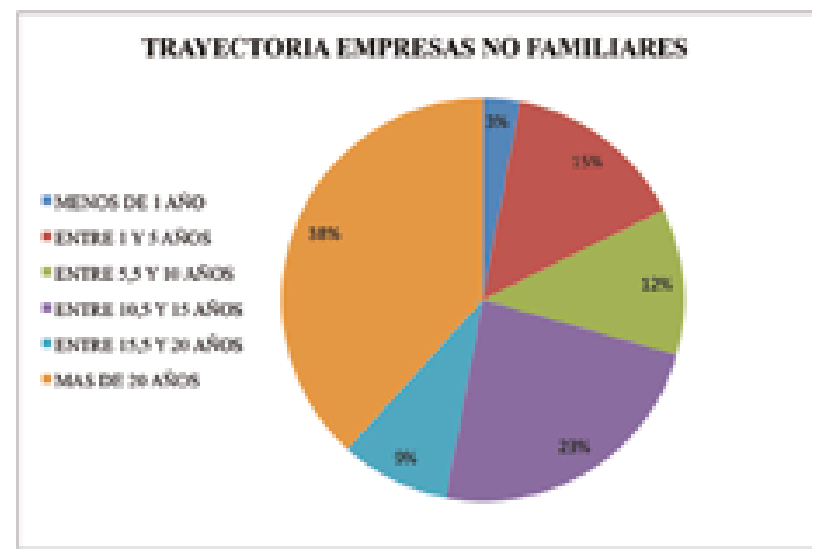

Fuente. Elaboración propia.

Figura 4. Trayectoria empresas familiares

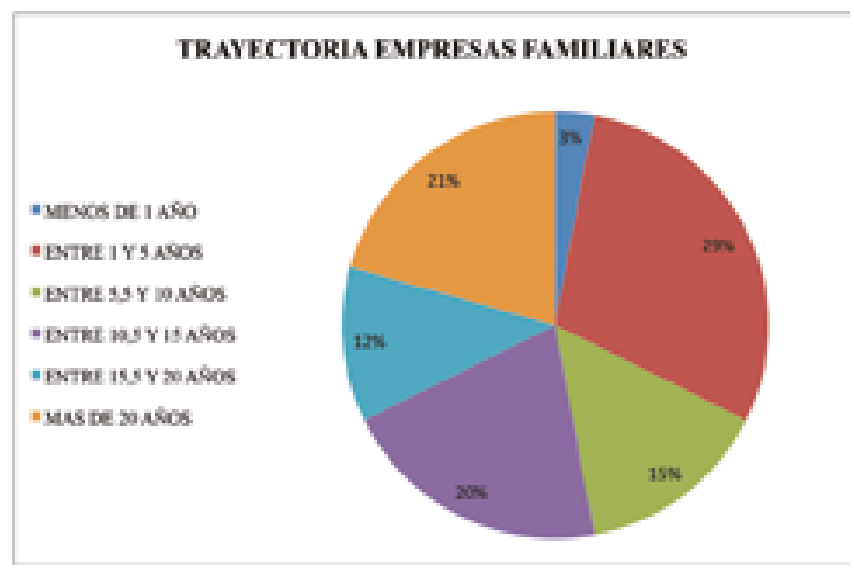

Fuente. Elaboración propia.

Para finalizar, en la caracterización de las empresas encuestadas se relaciona la distribución según el tamaño de la organización, de acuerdo con las figuras 5 y 6 se puede observar que la mayoría de las empresas que conforman la muestra de empresas no familiares son pequeñas $(35 \%)$ mientras que la mayoría de empresas que conforman las empresas familiares son micro (56\%); asimismo, las grandes empresas en la categoría no familiares representa el $18 \%$ de la muestra, a diferencia del $3 \%$ que representan en la categoría familiares. 
Figura 5. Tamaño empresas no familiares

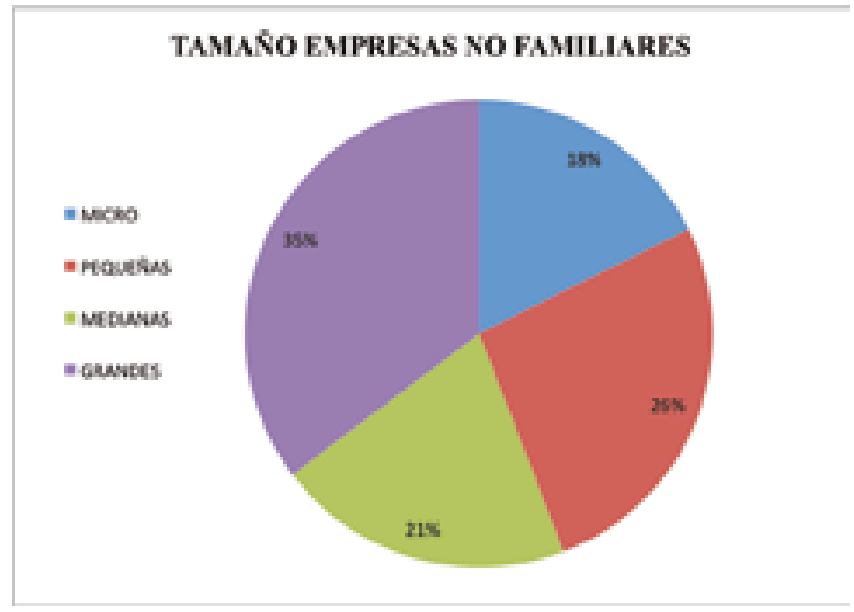

Fuente. Elaboración propia.

Figura 6. Tamaño empresas familiares

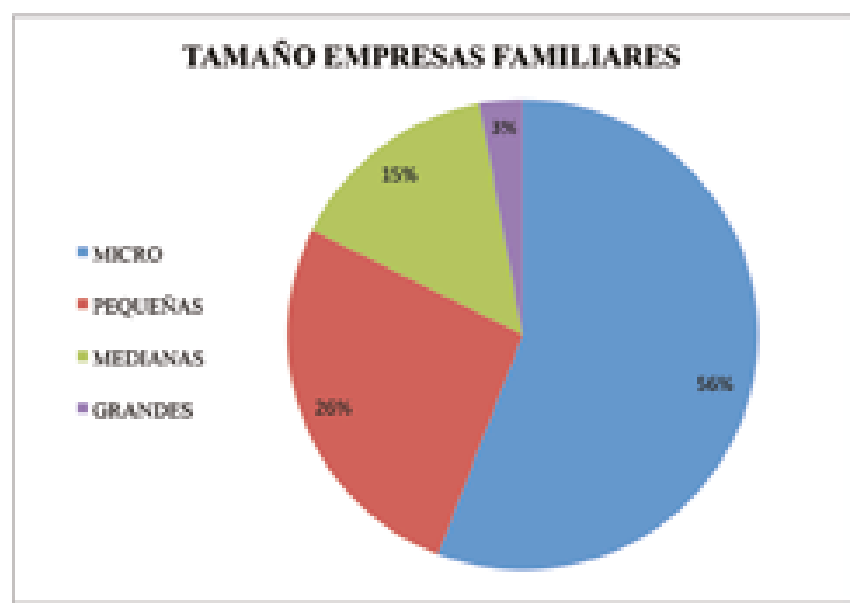

Fuente. Elaboración propia.

A continuación, se relacionan los cálculos econométricos mediante la implementación del modelo de regresión lineal simple entre las dimensiones de la capacidad dinámica de absorción del conocimiento para cada tipo de empresas, con la finalidad de conocer la incidencia y la relación que poseen entre las mismas para así posteriormente realizar la comparación entre empresas familiares y no familiares. Se realizó con anticipación a la aplicación del modelo econométrico un promedio ponderado de cada una de las dimensiones, con la finalidad de obtener un nuevo indicador para cada dimensión a través de la suma de cada uno de los ítems (pregun- 
tas) que contiene cada dimensión y la división entre el mismo número de ítems, esto para cada una de las etapas de la capacidad dinámica de absorción del conocimiento.

En primera instancia se presentan los resultados de los cálculos realizados entre las dimensiones de las empresas familiares, después se presentan los resultados de los cálculos realizados entre las dimensiones de las empresas no familiares para así poder concretar su debida comparación.

\section{Empresas familiares}

En la tabla 3 se muestran los resultados de los cálculos de correlaciones entre las dimensiones de las CDAC de las empresas familiares.

Tabla 3. Matriz de correlaciones entre las dimensiones en empresas familiares

\begin{tabular}{|c|c|c|c|c|c|}
\hline & & ADQUISICIÓN & ASIMILACIÓN & TRANSFORMACIÓN & EXPLOTACIÓN \\
\hline \multirow{3}{*}{$\begin{array}{l}\text { ADQUISI- } \\
\text { CIÓN }\end{array}$} & $\begin{array}{l}\text { Correlación } \\
\text { de Pearson }\end{array}$ & 1 &, $770^{* *}$ &, $784^{* *}$ &, $794^{* *}$ \\
\hline & $\begin{array}{l}\text { Sig. } \\
\text { (bilateral) }\end{array}$ & &, 000 &, 000 &, 000 \\
\hline & $\mathrm{N}$ & 34 & 34 & 34 & 34 \\
\hline \multirow{3}{*}{$\begin{array}{l}\text { ASIMILA- } \\
\text { CIÓN }\end{array}$} & $\begin{array}{l}\text { Correlación } \\
\text { de Pearson }\end{array}$ &, $770^{* *}$ & 1 &, $752^{* *}$ &, $828^{* *}$ \\
\hline & $\begin{array}{l}\text { Sig. } \\
\text { (bilateral) }\end{array}$ &, 000 & &, 000 &, 000 \\
\hline & $\mathrm{N}$ & 34 & 34 & 34 & 34 \\
\hline \multirow{3}{*}{$\begin{array}{l}\text { TRANSFOR- } \\
\text { MACIÓN }\end{array}$} & $\begin{array}{l}\text { Correlación } \\
\text { de Pearson }\end{array}$ &, $784^{* *}$ &, $752^{* *}$ & 1 &, $855^{* *}$ \\
\hline & $\begin{array}{l}\text { Sig. } \\
\text { (bilateral) }\end{array}$ &, 000 &, 000 & &, 000 \\
\hline & $\mathrm{N}$ & 34 & 34 & 34 & 34 \\
\hline \multirow{3}{*}{$\begin{array}{l}\text { EXPLOTA- } \\
\text { CIÓN }\end{array}$} & $\begin{array}{l}\text { Correlación } \\
\text { de Pearson }\end{array}$ &, $794^{* *}$ &, $828^{* *}$ &, $855^{* *}$ & 1 \\
\hline & $\begin{array}{l}\text { Sig. } \\
\text { (bilateral) }\end{array}$ &, 000 &, 000 &, 000 & \\
\hline & $\mathrm{N}$ & 34 & 34 & 34 & 34 \\
\hline
\end{tabular}

Fuente. Elaboración propia.

La correlación de Pearson es una medida lineal entre dos variables cuantitativas aleatorias que ayuda a determinar la correlación existente entre dos variables, para el caso de las empresas familiares los resultados de este análisis estadístico fueron los siguientes: entre las dimensiones Adquisición - Asimilación da un valor positivo de .770, entre las dimensiones Asimilación - Transformación dio un valor positivo de .752 y, por último, entre las dimensiones Transformación - Explotación dio un valor positivo de .855 . El valor positivo entre estas variables en cuanto a la correlación de Pearson nos indica que existe una correlación positiva entre cada una de ellas conforme a la secuencia de las dimensiones de la capacidad dinámica de absorción. 
La tabla 4 muestra el resumen de los modelos de regresión lineal realizados a las dimensiones.

En el modelo 1 se toma como variable independiente o predictora a la dimensión de la capacidad de absorción del conocimiento de las empresas familiares Adquisición, y como variable dependiente a la dimensión de Asimilación. Con un nivel de confianza del $95 \%$ con relación a la prueba " $t$ " student la variable predictora Adquisición sí es estadísticamente significativa para explicar los movimientos de la variable dependiente Asimilación con un grado de explicación del 59.3\%.

Tabla 4. Resultados de modelos econométricos en empresas familiares

\begin{tabular}{|c|c|c|c|c|c|c|c|c|c|}
\hline \multicolumn{5}{|c|}{ Resumen del modelo } & \multicolumn{5}{|c|}{ Coeficientes } \\
\hline & \multirow[t]{2}{*}{ Modelo } & \multirow[t]{2}{*}{$\mathrm{R}$} & \multirow{2}{*}{$\begin{array}{c}\mathrm{R} \\
\text { cuadrado }\end{array}$} & \multirow{2}{*}{$\begin{array}{l}\text { R cuadrado } \\
\text { corregido } \\
\text { B }\end{array}$} & \multicolumn{2}{|c|}{$\begin{array}{l}\text { Coeficientes no } \\
\text { estandarizados }\end{array}$} & \multirow[t]{2}{*}{$\begin{array}{c}\text { Coeficientes } \\
\text { tipificados }\end{array}$} & \multirow[t]{2}{*}{$\mathrm{t}$} & \multirow[t]{2}{*}{ Sig. } \\
\hline & & & & & Error típ. & Beta & & & \\
\hline 1 & $\begin{array}{l}\text { Variable } \\
\text { Independiente: } \\
\text { Adquisición } \\
\text { Variable } \\
\text { dependiente: } \\
\text { Asimilación } \\
\text { (Constante) }\end{array}$ &, $770^{\mathrm{a}}$ &, 593 &, 581 & $\begin{array}{l}1,004 \\
, 382\end{array}$ & $\begin{array}{l}, 147 \\
, 493\end{array}$ &, 770 & $\begin{array}{c}6,832 \\
, 775\end{array}$ & $\begin{array}{l}, 000 \\
, 444\end{array}$ \\
\hline 2 & $\begin{array}{l}\text { Variable } \\
\text { Independiente: } \\
\text { Asimilación } \\
\text { Variable } \\
\text { dependiente: } \\
\text { Transformación } \\
\text { (Constante) }\end{array}$ &, $752^{\mathrm{a}}$ &, 566 &, 552 & $\begin{array}{l}, 719 \\
1,141\end{array}$ & $\begin{array}{l}, 111 \\
, 424\end{array}$ &, 752 & $\begin{array}{l}6,458 \\
2,692\end{array}$ & $\begin{array}{l}, 000 \\
, 011\end{array}$ \\
\hline 3 & $\begin{array}{l}\text { Variable } \\
\text { Independiente: } \\
\text { Transformación } \\
\text { Variable } \\
\text { dependiente: } \\
\text { Explotación } \\
\text { (Constante) }\end{array}$ &, $855^{\mathrm{a}}$ &, 731 &, 723 & $\begin{array}{l}, 895 \\
, 121\end{array}$ & $\begin{array}{l}, 096 \\
, 375\end{array}$ &, 855 & $\begin{array}{r}9,327 \\
, 324\end{array}$ & $\begin{array}{l}, 895 \\
, 748\end{array}$ \\
\hline
\end{tabular}

Fuente. Elaboración propia.

Cabe mencionar que el coeficiente presenta un signo positivo, por lo tanto cualquier incremento en la dimensión de Adquisición afectará a la dimensión de Asimilación en forma positiva. Cuando la variable independiente tiende a cero, la variable dependiente tiene un valor de .382 , cuando la variable independiente incrementa una unidad porcentual la variable dependiente tiende a 1.004.

En el modelo 2, se toma como variable independiente o predictor a la dimensión de Asimilación y como variable dependiente a la dimensión de Transformación. Con un nivel de confianza del $95 \%$ con relación a la prueba " $t$ " student la variable predictora Asimilación sí es estadísticamente significativa para explicar los movimientos de la variable dependiente Transformación con un grado de explicación del 56.6\%. El coeficiente presenta un signo positivo, por lo tanto cualquier incremento en la dimen- 
sión de Asimilación afectará a la dimensión de Transformación en forma positiva. Cuando la variable independiente tiende a cero, la variable dependiente posee un valor de 1.141, cuando la variable independiente incrementa una unidad porcentual la variable dependiente tiende a .719 .

Por último, en el modelo 3 se toma como variable independiente o predictora a la dimensión de Transformación y como variable dependiente a la dimensión de Explotación. Con un nivel de confianza del 95\% con base en la prueba " $t$ " student la variable predictora Transformación sí es estadísticamente significativa para explicar los movimientos de la variable dependiente Explotación con un grado de explicación del 73.1\%. En este modelo nuevamente el coeficiente presenta un signo positivo, por lo tanto cualquier incremento en la dimensión de Transformación afectará a la dimensión de Explotación en forma positiva. Cuando la variable independiente tiende a cero, la variable dependiente tiene un valor de .121, cuando la variable independiente incrementa una unidad porcentual la variable dependiente tiende a .895 .

\section{Empresas no familiares}

Tabla 5. Matriz de correlaciones entre las dimensiones en empresas no familiares

\begin{tabular}{|c|c|c|c|c|c|}
\hline & & Adquicisión & Asimilación & Transformación & Explotación \\
\hline \multirow{3}{*}{ ADQUICISIÓN } & $\begin{array}{l}\text { Correlación } \\
\text { de Pearson }\end{array}$ & 1 &, $719^{* *}$ &, $596^{* *}$ &, $576^{* *}$ \\
\hline & $\begin{array}{l}\text { Sig. } \\
\text { (bilateral) }\end{array}$ & &, 000 &, 000 &, 000 \\
\hline & $\mathrm{N}$ & 34 & 34 & 34 & 34 \\
\hline \multirow{3}{*}{ ASIMILACIÓN } & $\begin{array}{l}\text { Correlación } \\
\text { de Pearson }\end{array}$ &, $719^{* *}$ & 1 &, $659^{* *}$ &, $673^{* *}$ \\
\hline & $\begin{array}{l}\text { Sig. } \\
\text { (bilateral) }\end{array}$ &, 000 & &, 000 &, 000 \\
\hline & $\mathrm{N}$ & 34 & 34 & 34 & 34 \\
\hline \multirow{3}{*}{ TRANSFORMACIÓN } & $\begin{array}{l}\text { Correlación } \\
\text { de Pearson }\end{array}$ &, $596^{* *}$ &, $659^{* *}$ & 1 &, $473^{* * *}$ \\
\hline & $\begin{array}{l}\text { Sig. } \\
\text { (bilateral) }\end{array}$ &, 000 &, 000 & &, 005 \\
\hline & $\mathrm{N}$ & 34 & 34 & 34 & 34 \\
\hline \multirow{3}{*}{ EXPLOTACIÓN } & $\begin{array}{l}\text { Correlación } \\
\text { de Pearson }\end{array}$ &, $576^{* *}$ &, $673^{* * *}$ &, $473^{* *}$ & 1 \\
\hline & $\begin{array}{l}\text { Sig. } \\
\text { (bilateral) }\end{array}$ &, 000 &, 000 &, 005 & \\
\hline & $\mathrm{N}$ & 34 & 34 & 34 & 34 \\
\hline
\end{tabular}

**. La correlación es significativa al nivel 0,01 (bilateral).

Fuente. Elaboración propia. 
Los resultados en la correlación de Pearson para las empresas no familiares son de igual manera positivos, lo cual indican una correlación positiva entre las dimensiones de forma secuencial en la CDAC de las empresas no familiares, para el caso de las dimensiones Adquisición - Asimilación dio un valor de .719, entre las dimensiones Asimilación - Transformación dio un valor de $.659 \mathrm{y}$, por último, entre las dimensiones Transformación - Explotación dio un valor de .473.

La tabla 6 muestra el resumen de los modelos de regresión lineal realizados a las dimensiones de la capacidad de absorción del conocimiento de las empresas no familiares.

Tabla 6. Resultados de modelos econométricos en empresas no familiares

\begin{tabular}{|c|c|c|c|c|c|c|c|c|c|}
\hline \multicolumn{5}{|c|}{ Resumen del modelo } & \multicolumn{5}{|c|}{ Coeficientes } \\
\hline & \multirow{2}{*}{ Modelo } & \multirow{2}{*}{$\mathrm{R}$} & \multirow{2}{*}{$\begin{array}{c}\mathrm{R} \\
\text { cuadrado }\end{array}$} & \multirow{2}{*}{$\begin{array}{c}\mathrm{R} \\
\text { cuadrado } \\
\text { corregido } \\
\text { B }\end{array}$} & \multicolumn{2}{|c|}{$\begin{array}{l}\text { Coeficientes no } \\
\text { estandarizados }\end{array}$} & \multirow[t]{2}{*}{$\begin{array}{l}\text { Coeficientes } \\
\text { tipificados }\end{array}$} & \multirow{2}{*}{$\mathrm{t}$} & \multirow{2}{*}{ Sig. } \\
\hline & & & & & Error típ. & Beta & & & \\
\hline 1 & $\begin{array}{l}\text { Variable } \\
\text { Independiente: } \\
\text { Adquisición } \\
\text { Variable } \\
\text { dependiente: } \\
\text { Asimilación } \\
\text { (Constante) }\end{array}$ & ,694 & ,481 & ,465 & $\begin{array}{l}, 887 \\
, 631\end{array}$ & $\begin{array}{l}, 163 \\
, 654\end{array}$ & ,694 & $\begin{array}{l}5,449 \\
, 969\end{array}$ & $\begin{array}{l}, 000 \\
, 340\end{array}$ \\
\hline 2 & $\begin{array}{l}\text { Variable } \\
\text { Independiente: } \\
\text { Asimilación } \\
\text { Variable } \\
\text { dependiente: } \\
\text { Transformación } \\
\text { (Constante) }\end{array}$ &, $586^{\mathrm{a}}$ & ,343 & ,322 & $\begin{array}{l}, 437 \\
2,478\end{array}$ & $\begin{array}{l}, 107 \\
, 452\end{array}$ &, 586 & $\begin{array}{l}4,087 \\
5,482\end{array}$ & $\begin{array}{l}, 000 \\
, 000\end{array}$ \\
\hline 3 & $\begin{array}{l}\text { Variable } \\
\text { Independiente: } \\
\text { Transformación } \\
\text { Variable } \\
\text { dependiente: } \\
\text { Explotación } \\
\text { (Constante) }\end{array}$ &, $761^{\mathrm{a}}$ &, 580 &, 567 & $\begin{array}{l}1,005 \\
-, 307\end{array}$ & $\begin{array}{l}, 151 \\
, 656\end{array}$ & ,761 & $\begin{array}{l}6,645 \\
-, 468\end{array}$ & $\begin{array}{l}, 000 \\
, 643\end{array}$ \\
\hline
\end{tabular}

Fuente. Elaboración propia.

En el modelo 1 de las empresas no familiares se toma como variable independiente o predictora a la dimensión de Adquisición y como variable dependiente a la dimensión de Asimilación. Con un nivel de confianza del 95\% con base en la prueba “ $t$ " student la variable predictora Adquisición si es estadísticamente significativa para explicar los movimientos de la variable dependiente Asimilación con un grado de explicación del $48.1 \%$, el coeficiente presenta un signo positivo, por lo tanto cualquier incremento en la dimensión de Adquisición afectará a la dimensión de Asimilación en forma positiva. Cuando la variable independiente tiende a cero, la variable 
dependiente tiene un valor de .631 , cuando la variable independiente incrementa una unidad porcentual la variable dependiente tiende a .887 .

En el modelo 2 de las empresas no familiares se toma como variable independiente o predictora la dimensión de Asimilación y como variable dependiente a la dimensión de Transformación. Con un nivel de confianza del 95\% con base en la prueba " $t$ " student la variable predictora Asimilación sí es estadísticamente significativa para explicar los movimientos de la variable dependiente Transformación con un grado de explicación del 34.3\%. En este modelo el coeficiente presenta un signo positivo, por lo tanto cualquier incremento en la dimensión de Asimilación afectará a la dimensión de Transformación en forma positiva. Cuando la variable independiente tiende a cero, la variable dependiente posee un valor de 2.478 , cuando la variable independiente incrementa una unidad porcentual la variable dependiente tiende a .437 .

Y en el modelo 3 de las empresas no familiares se toma como variable independiente o predictora a la dimensión de Transformación y como variable dependiente a la dimensión de Explotación. Con un nivel de confianza del 95\% con base en la prueba " $t$ " student la variable predictora Transformación sí es estadísticamente significativa para explicar los movimientos de la variable dependiente Explotación con un grado de explicación del 58\%. En este modelo el coeficiente presenta un signo positivo, por lo tanto cualquier incremento en la dimensión de Transformación afectará a la dimensión de Explotación en forma positiva. Cuando la variable independiente tiende a cero, la variable dependiente tiene un valor de -.307, cuando la variable independiente incrementa una unidad porcentual la variable dependiente tiende a 1.005.

Los resultados anteriores permiten validar el constructo propuesto por Zahra y George (2002), teniendo en cuenta que tanto en la muestra de empresas familiares como de no familiares se observa una relación entre las dimensiones propuestas (adquisición, asimilación, transformación y explotación). Además, se puede afirmar que tanto las empresas familiares como las no familiares estudiadas presentan desarrollo de la capacidad de absorción. De acuerdo con la hipótesis inicial planteada 'las empresas familiares presentan baja capacidad de absorción de conocimiento frente a las empresas no familiares', se rechaza teniendo en cuenta que tanto las empresas familiares como no familiares presentan desarrollo de la capacidad de absorción.

Las empresas familiares a pesar de las dificultades que enfrentan al no tener totalmente definido un sistema de gobernabilidad y políticas institucionales o sustitución de relevo generacional sí implementa estrategias que permiten garantizar la captación de información del exterior, su asimilación, transformación de prácticas e incorporación en sus bienes y servicios. 


\section{Conclusiones y recomendaciones}

La capacidad dinámica de absorción del conocimiento es uno de los factores más importantes en una empresa, ya que a través de sus dimensiones agregan valor a las prácticas empresariales.

En cuanto a los resultados obtenidos en los cálculos estadísticos, ambos tipos de empresas (familiares y no familiares) las variables independientes introducidas en los modelos de regresión lineal son estadísticamente significativas para explicar los movimientos de las variables dependientes, es decir, en ambos tipos de empresas existe una relación o dependencia entre la secuencia de las dimensiones de la CDAC positiva. Esto quiere decir que el instrumento utilizado tiene un alto grado de medición para la CDAC creando nuevos indicadores para calcular cada una de las dimensiones que la conforman con base en las afirmaciones utilizadas en el instrumento. En relación con la $\mathrm{R}$ cuadrada obtenida en los modelos, son las empresas familiares en comparación a las no familiares las que tienen mayor dependencia o explicación de su comportamiento con resultados de $59.3 \%, 56.6 \%$ y $71.3 \%$ en cuanto a las combinaciones basadas al ciclo de la CDAC, según Zahra y George (2002), que consta en 4 fases: Adquisición, Asimilación, Transformación y Explotación. Los resultados de los coeficientes de correlación de Pearson vienen a reafirmar que es en las empresas familiares donde existe mayor correlación entre las dimensiones al también obtener los resultados más altos con valores de .770 para la relación Adquisición - Asimilación .752 para la combinación Asimilación - Transformación y un valor de .855 en la combinación Transformación - Explotación, esta última relación en la última dimensión es de suma importancia, ya que es la dimensión donde las ideas se convierten en hechos o productos, dando paso a la innovación en las empresas, por lo tanto estos resultados en este tipo de empresas son altamente satisfactorios, ya que en Colombia al igual que a nivel mundial más del $90 \%$ de las empresas conformadas están dentro del ámbito familiar.

Esto no quiere decir que las empresas no familiares hayan tenido resultados negativos o contradictorios, sino que no fueron tan estadísticamente significativos en comparación con las empresas familiares, dentro del modelo econométrico realizado obtuvieron niveles de explicación positivos con valores de $48.1 \%$, 34.3\% y 58\% en su $\mathrm{R}$ cuadrada. Una de las grandes diferencias encontradas en este tipo de empresa es que en el caso de las empresas no familiares cuando la dimensión de Transformación tiende a no verse incrementada o no experimenta un movimiento por la dimensión anterior, la dimensión siguiente que es Explotación tiende a moverse de forma negativa -3.07 unidades porcentuales.

Los resultados probablemente estén explicados en el manejo administrativo integrado en las empresas familiares, caracterizado por mantener un sistema flexible y autónomo en el trabajo con comunicación informal de acuerdo con lo expresado por Ronquillo (2006) lo que aporta mayor capacidad de adaptación a nuevas prácticas 
empresariales. Un resultado por destacar es que de acuerdo con el análisis estadístico de las variables de estudio las empresas familiares poseen mayor capacidad de explotar el conocimiento adquirido.

Estos resultados son de gran utilidad para la comunidad científica al ser la primera investigación realizada en esta ciudad bajo esta línea y temática de investigación se genera nuevo y productivo conocimiento de carácter científico, nuevos indicadores de la CDAC a través de un nuevo instrumento con la capacidad de medir la incidencia de las dimensiones de la CDAC en las empresas y la validación del constructo propuesto por Zahra y George (2002).

Se recomienda para posteriores investigaciones dentro del marco de la capacidad dinámica de absorción del conocimiento considerar una muestra más amplia para así obtener resultados más concretos y generalizados sobre las empresas tanto familiares y no familiares. A su vez, realizar de forma comparativa un contraste entre los resultados de esta investigación en el año 2018 con las nuevas investigaciones, donde se pueda determinar el transcurso de la incidencia de las dimensiones de las CDAC dentro de las empresas y así determinar un reflejo del progreso o retroceso de estas en las empresas de Bucaramanga, Santander, Colombia. De igual forma, realizar el mismo estudio en otras ciudades para conocer y determinar si son las empresas familiares las que poseen mayor relación entre las mismas dimensiones y mayor grado de explicación de la CDAC.

\section{Referencias}

Celeita, J. L. (2016). Estrategias empresariales en un modelo de procesos de negocio para aumentar la rentabilidad y garantizar la permanencia en el mercado de una compañía. (Tesis de pregrado). Universidad Militar Nueva Granada, Bogotá. Colombia.

Cohen, W.M. \& Levinthal, D.A. (1990). Absorptive Capacity: A New Learning Perspective on Learning and Innovation. Administrative Science Quarterly, $35(1), 128-152$.

Confecámaras. (2016). Nacimiento y supervivencia de las empresas en Colombia. Red de Cámaras de Comercio de Colombia.

Díaz, N. L., Aguiar, I., y De Saá, P. (2006). El conocimiento organizativo tecnológico y la capacidad de innovación. Evidencia para la empresa industrial española. Cuadernos de Economía y Dirección de la Empresa, (27), 33-60.

Filgueiras, M., Castro, M., y Rafull, I. (2013). Determinación de la capacidad de absorción: estudio de caso en la empresa Geysel. Ingeniería Energética, 34(3), $175-185$. 
Flor, M.L., Oltra, M.J., García, C. (2011). La relación entre la capacidad de absorción del conocimiento externo y la estrategia empresarial: un análisis exploratorio. Revista Europea de Dirección y Economía de la Empresa, 20(1), 69-88.

Fong, C. (2011). La teoría de los recursos y capacidades: Un enfoque contemporáneo en la gestión empresarial. Carta Económica Regional, 61, 27-31. (U. d. Guadalajara, Ed.).

Franco, M., y Urbano, D. (2010). El éxito de las pymes en Colombia: un estudio de casos en el sector salud. Estudios Gerenciales, 26(114), 77-96. doi: 10.1016/S01235923(10)70103-0.

Gallo, M. (2004). Empresa familiar. Ed. Praxis. Barcelona, España.

Gaitán, A., Castro, J. 2005. Sociedades de familia en Colombia. Bogotá, Colombia: Superintendencia de Sociedades.

García, V., Ruiz, M., López, M. E., y Plascencia, I. (2018). Diversidad y ubicuidad como determinantes de la complejidad económica de las empresas. XVI Congreso Internacional de Análisis Organizacional "Realidades Organizacionales MéxicoCanadienses: Construyendo Vínculos Teóricos Comunes”. Homenaje al Dr. Alain Chanlat. Montreal, Canadá.

González, C. H., \& Hurtado, A. (2014). Propuesta de un indicador de capacidad de absorción del conocimiento (Icac-Col): evidencia empírica para el sector servicios en Colombia. Revista de la Facultad de Ciencias Económicas: Investigación y Reflexión, 22(2), 29-46.

Herrera, O. B., Parra, M., Livscovsky, I., Ramos, P., \& Gallardo, D. (2017). Lifeways and territorial innovation: values and practices for promoting collective appropriation of territory. Community Development Journal, 1-19, doi: 10.1093/ cdj/bsx052.

Lane, P. J., Koka, B. R., \& Pathak, S. (2006). The reification of absorptive capacity: A critical review and rejuvenation of the construct. Academy of management review, 31(4), 833-863, doi: 10.5465/amr.2006.22527456.

Leal, A. L., y Albort, G. (2015). Capacidad de absorción del conocimiento y resultados de la innovación: Un estudio empírico en empresas españolas del sector de la automoción. Universitas: Gestão e TI, 5(2). 35-47. doi: 10.5102/un.gti.v5i2.3618.

Máynez, A. I., Cavazos, J., y Nuño, J. P. (2012). La influencia de la cultura organizacional y la capacidad de absorción sobre la transferencia de conocimiento tácito intra-organizacional. Estudios Gerenciales, 28(Ed.E.), 191-211.

Nonaka, I., y Takeuchi, H. (1995). The knowledge-creating company: How Japanese companies create the dynamics of innovation. Oxford University Press. 
Pavez, A. A. (2000). Modelo de implantación de gestión del conocimiento y tecnologías de información para la generación de ventajas competitivas. Universidad Técnica Federico Santa María. Valparaíso, Chile.

Peña, J. C., Bravo, S., Álvarez, F. A., y Pineda, D. A. (2011). Analysis of characteristics of business incubators in Colombia: a case study (Analisis de las características de las incubadoras de empresas en Colombia: un estudio de casos). Journal of Economics, Finance and Administrative Science, 16(30), 13-30.

Poza, E. (2004). Empresas familiares. Ciudad de México, México: Ed. Thompson.

Ronquillo, J. (2006). Administración básica de la empresa familiar. Ciudad de México, México: Ed. Panorama.

Serna, H. (2006). Empresas de familia. Gestión para su supervivencia. Bogotá, Colombia: Temis.

Suárez, J., y Ibarra, S. (2002). La teoría de los recursos y las capacidades un enfoque actual en la estrategia empresarial. Anales de los Estudios Económicos y Empresariales, 15, 63-89

Tzokas, N., Kim, Y. A., Akbar, H., \& Al-Dajani, H. (2015). Absorptive capacity and performance: The role of customer relationship and technological capabilities in high-tech SMEs. Industrial Marketing Management, 47, 134-142. doi: 10.1016/j. indmarman.2015.02.033.

Zahra, S. A., \& George, G. (2002). Absorptive capacity: A review, reconceptualization, and extension. Academy of Management Review, 27(2), 185-203. doi: 10.5465/ amr.2002.6587995. 